\title{
The rainbow vertex-index of complementary graphs*
}

\author{
Research Article
}

\begin{abstract}
Fengnan Yanling ${ }^{1 * *}$, Zhao Wang $^{1 * * *}$, Chengfu Ye $^{1 \S}$, Shumin Zhang ${ }^{1 \S \S}$
\end{abstract}
1. Qinghai Normal University

\begin{abstract}
A vertex-colored graph $G$ is rainbow vertex-connected if two vertices are connected by a path whose internal vertices have distinct colors. The rainbow vertex-connection number of a connected graph $G$, denoted by $\operatorname{rvc}(G)$, is the smallest number of colors that are needed in order to make $G$ rainbow vertex-connected. If for every pair $u, v$ of distinct vertices, $G$ contains a vertex-rainbow $u-v$ geodesic, then $G$ is strongly rainbow vertex-connected. The minimum $k$ for which there exists a $k$-coloring of $G$ that results in a strongly rainbow-vertex-connected graph is called the strong rainbow vertex number $\operatorname{srvc}(G)$ of $G$. Thus $\operatorname{rvc}(G) \leq \operatorname{srvc}(G)$ for every nontrivial connected graph $G$. A tree $T$ in $G$ is called a rainbow vertex tree if the internal vertices of $T$ receive different colors. For a graph $G=(V, E)$ and a set $S \subseteq V$ of at least two vertices, an $S$-Steiner tree or a Steiner tree connecting $S$ (or simply, an $S$-tree) is a such subgraph $T=\left(V^{\prime}, E^{\prime}\right)$ of $G$ that is a tree with $S \subseteq V^{\prime}$. For $S \subseteq V(G)$ and $|S| \geq 2$, an $S$-Steiner tree $T$ is said to be a rainbow vertex $S$-tree if the internal vertices of $T$ receive distinct colors. The minimum number of colors that are needed in a vertex-coloring of $G$ such that there is a rainbow vertex $S$-tree for every $k$-set $S$ of $V(G)$ is called the $k$-rainbow vertex-index of $G$, denoted by $r v x_{k}(G)$. In this paper, we first investigate the strong rainbow vertex-connection of complementary graphs. The $k$-rainbow vertex-index of complementary graphs are also studied.
\end{abstract}

2010 MSC: 05C15, 05C40

Keywords: Strong rainbow vertex-connection number, Complementary graph, Rainbow vertex $S$-tree, $k$-rainbow vertex-index

\section{Introduction}

The graphs considered in this paper are finite undirected and simple graphs. We follow the notation of Bondy and Murty [1], unless otherwise stated. For a graph $G$, let $V(G), E(G), n(G), m(G)$, and $\bar{G}$, respectively, be the set of vertices, the set of edges, the order, the size, and the complement graph of $G$.

\footnotetext{
* Supported by the National Science Foundation of China (No. 11161037, 11101232, 11461054) and the Science Found of Qinghai Province (No. 2014-ZJ-907).

** E-mail: fengnanyanlin@yahoo.com (Corresponding Author)

*** E-mail: wangzhao380@yahoo.com

$\S$ E-mail: yechf@qhnu.edu.cn

$\S \S$ E-mail: zhsm_0926@sina.com
} 
Let $G$ be a nontrivial connected graph on which an edge-coloring $c: E(G) \rightarrow\{1,2, \cdots, n\}, n \in \mathbb{N}$, is defined, where adjacent edges may be colored the same. A path is rainbow if no two edges of it are colored the same. An edge-coloring graph $G$ is rainbow connected if any two vertices are connected by a rainbow path. Clearly, if a graph is rainbow connected, it must be connected, whereas any connected graph has a trivial edge-coloring that makes it rainbow connected; just color each edge with a distinct color. Thus, in [4] L. Chen, X. Li, H. Lian defined the rainbow connection number of a connected graph $G$, denoted by $\operatorname{rc}(G)$, as the smallest number of colors that are needed in order to make $G$ rainbow connected. They showed that $r c(G) \geq \operatorname{diam}(G)$ where $\operatorname{diam}(G)$ denotes the diameter of $G$. For more results on the rainbow connection, we refer to the survey paper [2],[3],[4] and [12], and a new book [10] of $\mathrm{Li}$ and Sun.

In [8], Krivelevich and Yuster proposed the concept of rainbow vertex-connection. A vertex-colored graph $G$ is rainbow vertex-connected if two vertices are connected by a path whose internal vertices have distinct colors. The rainbow vertex-connection number of a connected graph $G$, denoted by $r v c(G)$, is the smallest number of colors that are needed in order to make $G$ rainbow vertex-connected. For more results on the rainbow vertex-connection, we refer to the survey paper [5] and [9]. An easy observation is that if $G$ is of order $n$, then $\operatorname{rvc}(G) \leq n-2$ and $\operatorname{rvc}(G)=0$ if and only if $G$ is a complete graph. Notice that $\operatorname{rvc}(G) \geq \operatorname{diam}(G)-1$ with equality if the diameter is 1 or 2 .

If for every pair $u, v$ of distinct vertices, $G$ contains a vertex-rainbow $u-v$ geodesic, then $G$ is strong rainbow vertex-connected. The definition of strongly rainbow vertex-connected was defined by $\mathrm{Li}$ et al. in [11]. The minimum $k$ for which there exists a $k$-coloring of $G$ that results in a strongly rainbow vertex-connected graph is called the strong rainbow vertex-connection number srvc $(G)$ of $G$. Thus $\operatorname{rc}(G) \leq \operatorname{srvc}(G)$ for every nontrivial connected graph $G$.

If $G$ is a nontrivial connected graph of order $n$ whose diameter is $\operatorname{diam}(G)$, then

$$
\operatorname{diam}(G)-1 \leq \operatorname{rvc}(G) \leq \operatorname{srvc}(G) \leq n-s,
$$

where $s$ denote the number of pendent vertices in $G$.

Proposition 1.1. Let $G$ be a nontrivial connected graph of order $n$. Then

(a) $\operatorname{srvc}(G)=0$ if and only if $G$ is a complete graph;

(b) $\operatorname{srvc}(G)=1$ if and only if $\operatorname{diam}(G)=2$ if and only if $\operatorname{rvc}(G)=1$.

A tree $T$ in $G$ is called a rainbow vertex tree if the internal vertices of $T$ receive different colors. For a graph $G=(V, E)$ and a set $S \subseteq V$ of at least two vertices, an $S$-Steiner tree or a Steiner tree connecting $S$ (or simply, an $S$-tree) is a such subgraph $T=\left(V^{\prime}, E^{\prime}\right)$ of $G$ that is a tree with $S \subseteq V^{\prime}$. For more problems on $S$-Steiner tree, we refer to [6] and [7].

For $S \subseteq V(G)$ and $|S| \geq 2$, an $S$-Steiner tree $T$ is said to be a rainbow vertex $S$-tree if the internal vertices of $T$ receive distinct colors. The minimum number of colors that are needed in an vertex-coloring of $G$ such that there is a rainbow vertex $S$-tree for every $k$-set $S$ of $V(G)$ is called the $k$-rainbow vertexindex of $G$, denoted by $r v x_{k}(G)$. The vertex-rainbow index of a graph was first defined by Yaping Mao in [13].

\section{The strong rainbow vertex-connection of complementary graphs}

In this section, we investigate the rainbow vertex-connection number of a graph $G$ according to some constraints to its complement $\bar{G}$. We give some conditions to guarantee that $\operatorname{srvc}(G)$ is bounded by a constant.

We investigate the rainbow vertex-connection number of connected complement graphs of graphs with diameter at least 3 . 
Theorem 2.1. If $G$ is a connected graph with $\operatorname{diam}(G) \geq 3$, then

$$
\operatorname{srvc}(\bar{G})= \begin{cases}1, & \text { if } \operatorname{diam}(G) \geq 4 \\ 2, & \text { if } \operatorname{diam}(G)=3\end{cases}
$$

Proof. We choose a vertex $x$ with $\operatorname{ecc}_{G}(x)=\operatorname{diam}(G)=d \geq 3$. Let $N_{G}^{i}(x)=\left\{v: d_{G}(x, v)=i\right\}$ where $0 \leq i \leq d$. So $N_{G}^{0}(x)=\{x\}, N_{G}^{1}(x)=N_{G}(x)$ as usual. Then $\bigcup_{0<i<d} N_{G}^{i}(x)$ is a vertex partition of $V(G)$ with $\left|N_{G}^{i}(x)\right|=n_{i}$. Let $A=\bigcup_{i \text { is even }} N_{G}^{i}(x), B=\bigcup_{i \text { is odd }} N_{G}^{i}(x)$. For example, see Figure 1, a graph with $\operatorname{diam}(G)=5$.

So, if $d=2 k(k \geq 2)$, then $A=\bigcup_{0 \leq i \leq d \text { is even }} N_{G}^{i}(x), B=\bigcup_{1 \leq i \leq d-1}$ is odd $N_{G}^{i}(x)$; if $d=2 k+1(k \geq$ 2) then $A=\bigcup_{0 \leq i \leq d-1}$ is even $N_{G}^{i}(x), \bar{B}=\bigcup_{1 \leq i \leq d}$ is odd $N_{G}^{i}(x)$. Then by the definition of complement graphs, we know that $\bar{G}[A](\bar{G}[B])$ contains a spanning complete $k_{1}$-partite subgraph(complete $k_{2}$-partite subgraph) where $k_{1}=\left\lceil\frac{d+1}{2}\right\rceil\left(k_{2}=\left\lceil\frac{d}{2}\right\rceil\right)$. For example, see Figure $1, \bar{G}[A]$ contains a spanning complete tripartite subgraph $K_{n_{0}, n_{2}, n_{4}}, \bar{G}[B]$ contains a spanning complete tripartite subgraph $K_{n_{1}, n_{3}, n_{5}}$.

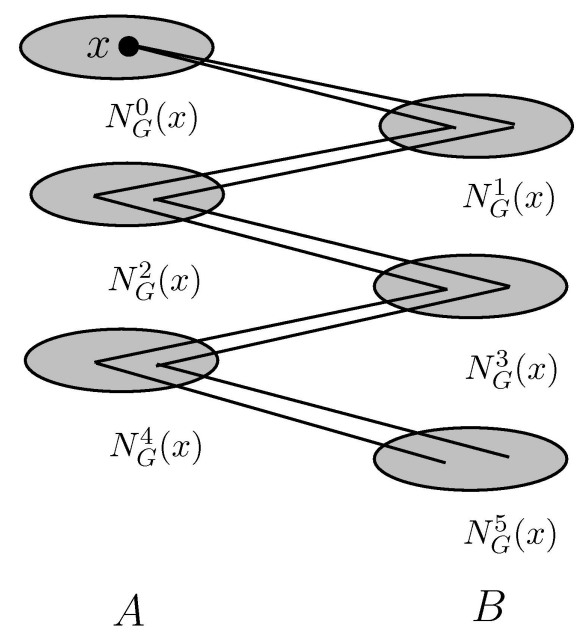

Figure 1. Graphs for the proof of Theorem 2.

First of all, we see that $\bar{G}$ must be connected, since otherwise, $\operatorname{diam}(G) \leq 2$, contradicting the condition $\operatorname{diam}(G) \geq 3$.

Case 1. $d \geq 5$.

In this case, $k_{1}, k_{2} \geq 3$. We will show that $\operatorname{diam}(\bar{G}) \leq 2$ in this case. For $u, v \in V(\bar{G})$, we consider the following cases:

Subcase 1.1. $u, v \in A$ or $u, v \in B$.

If $u, v \in A$, then $u, v$ is contained in the spanning complete $k_{1}$-partite subgraph of $\bar{G}[A]$. Thus $d_{\bar{G}}(u, v) \leq 2$. The same is true for $u, v \in B$.

Subcase 1.2. $u \in A$ and $v \in B$.

If $u=x, v \in B$, then $u$ is adjacent to all vertices in $\bar{G}[B] \backslash N_{G}^{1}(x)$. So $d_{\bar{G}}(u, v)=1$ for $v \in$ $\bar{G}[B] \backslash N_{G}^{1}(x)$. For $v \in N_{G}^{1}(x)$, let $P=u x_{3} v$, where $x_{3} \in N_{G}^{3}(x)$. Clearly, $d_{\bar{G}}(u, v)=2$.

If $u \neq x$, without loss of generality, we assume that $u \in N_{G}^{2}(x)$ and $v \in N_{G}^{1}(x)$. Let $Q=u x_{5} v$, where $x_{5} \in N_{G}^{5}(x)$. Thus $d_{\bar{G}}(u, v)=2$.

From the above, we conclude that $\operatorname{diam}(\bar{G}) \leq 2$. So, by Proposition $1(b)$, we have $\operatorname{srvc}(\bar{G})=1$. 
Case 2. $d=4$.

It is obvious that $A=N_{G}^{0}(x) \cup N_{G}^{2}(x) \cup N_{G}^{4}(x), B=N_{G}^{1}(x) \cup N_{G}^{3}(x)$. So $\bar{G}[A](\bar{G}[B])$ contains a spanning complete 3 -partite subgraph $K_{n_{0}, n_{2}, n_{4}}$ (complete bipartite subgraph $K_{n_{1}, n_{3}}$ ). So, we will show that $\operatorname{diam}(G) \leq 2$.

Subcase 2.1. $u, v \in A$ or $u, v \in B$.

If $u, v \in A$, then $u, v$ is contained in the spanning complete $k_{1}$-partite subgraph of $\bar{G}[A]$. Thus $d_{\bar{G}}(u, v) \leq 2$. If $u, v \in B$, then $u, v$ is contained in the spanning complete bipartite subgraph of $\bar{G}[B]$. Also we have $d_{\bar{G}}(u, v) \leq 2$.

Subcase 2.2. $u \in A$ and $v \in B$.

If $u=x, v \in B$, then $u$ is adjacent to all vertices in $N_{G}^{3}(x)$. For $v \in N_{G}^{1}(x)$, let $P=u x_{3} v$, where $x_{3} \in N_{G}^{3}(x)$. Clearly, $d_{\bar{G}}(u, v)=2$. So $d_{\bar{G}}(u, v) \leq 2$.

If $u \neq x$, then we assume that $u \in N_{G}^{2}(x)$ and $v \in N_{G}^{1}(x)$. Let $Q=u x_{4} v$, where $x_{4} \in N_{G}^{4}(x)$. Thus $d_{\bar{G}}(u, v)=2$. Suppose $u \in N_{G}^{4}(x)$ and $v \in N_{G}^{3}(x)$. Let $R=u x_{1} v$, where $x_{1} \in N_{G}^{1}(x)$. Thus $d \bar{G}_{\bar{G}}(u, v)=2$. If $u \in N_{G}^{2}(x)$ and $v \in N_{G}^{3}(x)$, then $S=u x v$ is a path of length 2. Then $\operatorname{diam}(G) \leq 2$. So, by Proposition 1 , we have $\operatorname{srvc}(\bar{G})=1$.

Case 3. $d=3$.

In this case, $A=N_{G}^{0}(x) \cup N_{G}^{2}(x), B=N_{G}^{1}(x) \cup N_{G}^{3}(x)$. So $\bar{G}[A]$ contains a spanning complete bipartite subgraph $K_{n_{0}, n_{2}}$. So, we give $\bar{G}$ a vertex-coloring as follows: color vertex $x$ with 1 and color all vertices of $N_{G}^{3}(x)$ with 2 . It is easy to see that for any $u \in N_{G}^{2}(x), v \in N_{G}^{1}(x)$, there is a rainbow $\{1,2\}$ path connecting them in $\bar{G}$. So $\operatorname{srvc}(\bar{G})=2$ in this case.

For the case of $\operatorname{diam}(G)=2, \operatorname{srvc}(\bar{G})$ can be very large since $\operatorname{diam}(\bar{G})$ may be very large. For example, let $G=K_{n} \backslash E\left(C_{n}\right)$, where $C_{n}$ is a cycle of length $n$ in $K_{n}$. Then $\bar{G}=C_{n}$ and $\operatorname{srvc}(\bar{G}) \geq$ $\operatorname{diam}(\bar{G})-1=\left\lceil\frac{n}{2}\right\rceil-1$ by $(1)$.

\section{The $k$-rainbow vertex-index of complete multipartite graphs}

Theorem 3.1. Let $K_{n_{1}, n_{2}, \cdots n_{l}}$ be a complete multipartite graph. If $k<2 \ell$, then $r v x_{k}=1$; If $k \geq 2 \ell$, then rvx $_{k}=2$. Where $S=\left\{v_{1}, v_{2}, \cdots v_{k}\right\}$ (that is the rainbow $S$-tree we choose) and $V_{n_{i}},(1 \leq i \leq l)$ are the vertices of the partition of $K_{n_{1}, n_{2}, \cdots n_{\ell}}$.

Proof. If $k<2 \ell$, then we can find a partition $V_{n_{i}},(1 \leq i \leq l)$ of $K_{n_{1}, n_{2}, \cdots n_{l}}$ with $V_{n_{i}} \cap S \leq 1$. If $V_{n_{i}} \cap S=\emptyset$, then we can choose a vertex $v \in V_{n_{i}}$ as the root vertex of the rainbow $S$ tree and all the other vertices are leaves. So $\operatorname{rvx}_{k}\left(K_{n_{1}, n_{2}, \cdots n_{\ell}}\right)=1$. If $V_{n_{i}} \cap S=1$, then we choose the vertex $v \in V_{n_{i}}$ as the root vertex of the rainbow $S$ tree, and all the other vertices are leaves. So $r v x_{k}\left(K_{n_{1}, n_{2}, \cdots n_{\ell}}\right)=1$.

If $k \geq 2 \ell$ and there exists $V_{n_{i}}$ such that $\left|S \cap V_{n_{i}}\right| \leq 1$, then we can choose the vertex $v$ in $V_{n_{i}}$ as the root of the rainbow tree and all the other vertices are the leaves the same as when $k<2 \ell$. So $\operatorname{rvx}_{k}\left(K_{n_{1}, n_{2}, \cdots n_{l}}\right)=1$

Suppose $k \geq 2 \ell$ and $\left|S \cap V_{n_{i}}\right| \geq 2$ for any $V_{n_{i}}$. Now we give a rainbow vertex-coloring as follows.

$$
c\left(V_{n_{i}}\right)=\left\{\begin{array}{l}
1, \text { if } 1 \leq i \leq \ell-1 \\
2, \text { if } i=\ell
\end{array}\right.
$$

Next we prove it is a $k$-rainbow vertex-coloring. Choose one vertex $v$ in $V_{n_{\ell}}$ as the root vertex of the rainbow tree. Obviously $v$ is adjacent to all the vertices in $V_{n_{1}} \cap S, V_{n_{2}} \cap S, \ldots V_{n_{\ell-1}} \cap S$. Then choose a vertex in $v^{\prime} \in V_{n_{1}}$. Since $v^{\prime}$ is adjacent to all the remaining vertices in $V_{n_{\ell}} \cap S$, one can prove that the tree is rainbow $S$-tree. 


\section{The $k$-rainbow vertex-index of complementary graphs}

Theorem 4.1. If $G$ is a connected graph with $\operatorname{diam}(G) \geq 3$, then $\operatorname{rvx}_{k}(\bar{G}) \leq 2$ and the bound is tight.

Proof. We choose a vertex $x$ with $\operatorname{ecc}_{G}(x)=\operatorname{diam}(G)=d \geq 3$ as Figure 1. Then $\bar{G}[A](\bar{G}[B])$ contains a spanning complete $k_{1}$-partite subgraph (complete $k_{2}$-partite subgraph). If the rainbow $S$-tree contains in $\bar{G}[A](\bar{G}[B])$, then $\operatorname{rvx}_{k}(\bar{G}) \leq 2$ by Theorem 3.1. Now we consider the rainbow $S$-tree does not contain in $\bar{G}[A]$ or $\bar{G}[B]$. If $S \cap N_{G}^{1}(G)=\emptyset$, then we choose $x$ as the root vertex, and all the other vertices are the leaves. So one can prove that there is a rainbow $S$-tree. Suppose $S \cap N_{G}^{1}(G) \neq \emptyset$. Now we give a rainbow vertex-coloring as follows.

$$
\left\{\begin{array}{l}
c(x)=1, \\
c(v)=2, \quad v \in V(G) \backslash x .
\end{array}\right.
$$

We choose the vertex $x$ as the root of the rainbow tree. We know $x$ is adjacent to all the vertices in $N_{G}^{j}(x) \cap S,(j \in\{2,3,4, \cdots\})$, and there must be a $v \in N_{G}^{j}(x),(j \in\{2 m+1$ and $m \geq 1\})$ such that $v$ is adjacent to $N_{G}^{1}(x) \cap S$. one can prove that the tree is rainbow $S$-tree.

Let $G$ is a connected graph of $\operatorname{diam}(G)=3$. We have $\operatorname{rvx}_{k}(\bar{G})=2$, so the bound is tight.

Acknowledgement: The authors are very grateful to the referees' valuable comments and suggestions, which helped greatly to improve the presentation of this paper.

\section{References}

[1] J. A. Bondy and U. S. R. Murty, Graph theory, GTM 244, Springer, 2008.

[2] G. Chartrand, G. L. Johns, K. A. McKeon and P. Zhang, Rainbow connection in graphs, Math. Bohem., 133, 85-98, 2008.

[3] G. Chartrand, G. L. Johns, K. A. McKeon and P. Zhang, The rainbow connectivity of a graph, Networks, 54(2), 75-81, 2009.

[4] L. Chen, X. Li and H. Lian, Nordhaus-Gaddum-type theorem for rainbow connection number of graphs, Graphs Combin., 29(5), 1235-1247, 2013.

[5] L. Chen, X. Li and M. Liu, Nordhaus-Gaddum-type theorem for the rainbow vertex connection number of a graph, Utilitas Math., 86, 335-340, 2011.

[6] X. Cheng and D. Du, Steiner trees in industry, Kluwer Academic Publisher, Dordrecht, 2001.

[7] D. Du and X. Hu, Steiner tree problems in computer communication networks, World Scientific, 2008.

[8] M. Krivelevich and R. Yuster, The rainbow connection of a graph is (at most) reciprocal to its minimum degree three, J. Graph Theory, 63(3), 185-191, 2010.

[9] X. Li and Y. Shi, On the rainbow vertex-connection, Discuss. Math. Graph Theory, 33(2), 307-313, 2013.

[10] X. Li and Y. Sun, Rainbow connections of graphs, SpringerBriefs in Math., Springer, New York, 2012.

[11] X. Li, Y. Mao and Y. Shi, The strong rainbow vertex-connection of graphs, Utilitas Math., 93, 213$223,2014$.

[12] X. Li, Y. Shi and Y. Sun, Rainbow connections of graphs-A survey, Graphs Combin., 29(1), 1-38, 2013.

[13] Y. Mao, The vertex-rainbow index of a graph, arXiv:1502.00151v1 [math.CO], 31 Jan 2015.

[14] E. A. Nordhaus and J. W. Gaddum, On complementary graphs, Amer. Math. Monthly, 63, 175-177, 1956. 